\title{
Individual Differences in the Effects of Physical Activity on Cognitive Function in People with Mild to Moderate Dementia
}

\author{
Iris L. Uijen ${ }^{\mathrm{a}, 1}$, Justine A. Aaronson ${ }^{\mathrm{b}, \mathrm{c}, 1}$, Esther G.A. Karssemeijer ${ }^{\mathrm{a}}$, Marcel G.M. Olde Rikkert ${ }^{\mathrm{a}}$ \\ and Roy P.C. Kessels ${ }^{\mathrm{c}, \mathrm{d}, \mathrm{e}, *}$ \\ ${ }^{a}$ Department of Geriatric Medicine \& Radboudumc Alzheimer Center, Radboud University Medical Center, \\ Nijmegen, The Netherlands \\ ${ }^{\mathrm{b}}$ OLVG Hospital, Amsterdam, The Netherlands \\ ${ }^{\mathrm{c}}$ Department of Medical Psychology \& Radboudumc Alzheimer Center, Radboud University Medical Center, \\ Nijmegen, The Netherlands \\ ${ }^{\mathrm{d}}$ Donders Institute for Brain, Cognition and Behaviour, Radboud University, Nijmegen, The Netherlands \\ ${ }^{\mathrm{e}}$ Vincent van Gogh Institute for Psychiatry, Venray, The Netherlands
}

Handling Associate Editor: Olivia Küster

Accepted 13 January 2020

\begin{abstract}
The aim of this study was to investigate whether the effect of physical activity on cognitive function in persons with dementia is moderated by patient characteristics as Apolipoprotein E and dementia type. We included 101 individuals with dementia and calculated the reliable change index to determine the change in global cognition, executive function, episodic memory, working memory, and processing speed before and after a 12-week exercise training. We found a higher treatment-related benefit in episodic memory in persons with non-Alzheimer's disease compared to persons with Alzheimer's disease, and in executive function in individuals with better baseline cognitive function.
\end{abstract}

Keywords: $A P O E \varepsilon 4$, cognitive function, dementia, physical activity, reliable change index

\section{INTRODUCTION}

There is an increasing interest in the potential of exercise as an intervention for cognitive decline in people with dementia. To date, the results from exercise studies are inconclusive [1,2], which may imply that physical activity is not a one-size-fits-all

\footnotetext{
${ }^{1}$ These authors contributed equally to this work.

${ }^{*}$ Correspondence to: Roy P.C. Kessels, PhD, Department of Medical Psychology, Internal Post 925, PO Box 9101, 6500 HB Nijmegen, The Netherlands. E-mail: roy.kessels@ radboudumc.nl.
}

intervention. The effects of exercise may be moderated by patient characteristics, such as the $\varepsilon 4$ allele of the gene Apolipoprotein E (APOE) and type of dementia. Previous studies with $A P O E \varepsilon 4$ status as moderator showed mixed results $[3,4]$. As for the type of dementia, the effect has not been investigated to date. We hypothesize that persons with vascular dementia would benefit most from aerobic exercise intervention as it improves vascular function and modifies key cardiometabolic risk factors [5]. Studies on these moderating effects are of great importance, as they can help determine who will 
benefit most from physical activity interventions and can help develop individualized training programs accordingly. The objective of this secondary analysis of a previously published randomized controlled trial (RCT) was to examine whether the effect of physical activity on cognitive function in persons with mild to moderate dementia is moderated by patient characteristics [6]. It was hypothesized that combined physical and cognitive exergame training would result in larger improvements in cognitive function than physical training alone. There is growing evidence for this in healthy older adults and in people with mild cognitive impairment, but this effect is less clear in persons with dementia [7]. In the $\mathrm{RCT}$, an extensive neuropsychological test battery was administered to evaluate the cognitive domains of executive function, episodic memory, working memory, and processing speed, as we expected a differential effect of exercise interventions on these domains [6]. The main outcome was that both physical intervention groups (combined cognitivephysical training and aerobic training) improved equally on psychomotor speed, compared to an active control group.

\section{METHODS}

\section{Participants, procedure, and outcome measures}

This study used data from a single-blind RCT investigating the effect of a 12-week combined cognitive-physical training on cognitive functioning compared to a single aerobic training and an active control group. A more detailed description of the methods and the group results have been presented previously [8]. In total, 115 persons with mild to moderate dementia, aged 60 years or above, were recruited through the memory clinic of the Radboudumc Alzheimer Center, day-care centers, advertisements in newspapers and word of mouth. $A P O E$ genotype, severity of cognitive decline, and type of dementia were determined at baseline. Saliva samples were analyzed using real-time polymerase chain reaction to determine $A P O E$ genotype. The type of dementia was classified into vascular dementia, Alzheimer's disease, mixed type, and unspecified using medical records. The severity of cognitive decline was assessed by the Mini-Mental State Examination (MMSE). Participants were randomized in to one of the two intervention groups (single aerobic training or combined cognitive-physical training), or an active control condition. The training in the com- bined cognitive-physical training group consisted of a cycling training on a stationary bike that was connected to a video screen. On this video screen, participants followed a route through a virtual environment and simultaneously performed cognitive tasks. Participants in the single aerobic group performed the same cycling training on a stationary bike, without connection to a video screen. Participants in the control group received stretching and toning exercises. The training sessions were given on an individual basis and all participants trained three times a week (30-40 min) for 12 weeks. Before and after the intervention period cognition was assessed using an extensive neuropsychological test battery that covered the following four cognitive domains: executive function (Stroop Color Word Test card III, Trail Making Test part B, Letter fluency and the Rule Shift Cards Test from the Behavioural Assessment of the Dysexecutive Syndrome), working memory (Digit Span from the Wechsler Adult Intelligence Scale-Third Edition and Spatial Span from the Wechsler Memory Scale-Third Edition), episodic memory (Location Learning Test-Revised), and processing speed (Trail Making Test part A and Stroop Color Word Test cards I \& II). A full description of the neuropsychological test battery can be found in the protocol paper [8].

The study was conducted in compliance with Declaration of Helsinki ethical standards and was approved by the Medical Ethical Committee of the Radboud University Medical Center. The study is registered at the Dutch Trial Register with identification number NTR5581. All participants gave written informed consent prior to screening.

\section{Statistical analysis}

To examine the individual effects of the intervention on cognition, the reliable change index (RCI) was calculated [9]. The RCI determines whether the difference between the pre-test and post-test score of an individual is statistically significant and therefore if the individual significantly improves or worsens, taking measurement error, test-retest reliability, and treatment-nonspecific changes in the control group into account. RCIs were calculated for global cognition (an overall domain score in which all four cognitive domain scores were averaged) and for all four cognitive domains separately for all individual participants in the two intervention groups. The control group was used as reference group for calculating the standard deviation and correlation between the 
pre-test and post-test scores. The following formula was used to calculate the RCI:

$$
\frac{\left(X_{2}-X_{1}\right)-\left(M_{2}-M_{1}\right)}{\sqrt{2\left(s d \sqrt{1-r_{x x}}\right)^{2}}}
$$

where $X_{2}$ is the post-test score and $X_{1}$ the pre-test score of the participant, $M_{2}$ is the mean post-test score and $M_{1}$ is the mean pre-test score of the control group, $s d$ the standard deviation of the control group on the pre-test, and $r_{x x}$ the correlation between the pre-test and post-test score in the control group. An RCI larger than 1.645 ( $\alpha=0.05$; one-tailed) indicates a significant improvement, referred to as a responder.

To examine whether the selected characteristics were associated with the effect of physical activity on cognitive function, multiple regression analyses were performed for the global cognition score and all four cognitive domains separately, with the RCI as dependent variable. Given the number of participants, we a priori decided to include four predictor variables, of which two possible moderators (type of dementia and $A P O E$ genotype) and two control variables (age and MMSE score). The vast majority of the participants had Alzheimer's disease, therefore the groups created for type of dementia were: Alzheimer's disease and other types (vascular dementia, mixed type, and unspecified). $\alpha$ was set at 0.05 .

\section{RESULTS}

Fourteen participants (12\%) did not complete the training and were excluded from the analyses. A full description of the reasons for drop-out has been provided previously [6]. Main reasons for drop-out were comorbid medical or physical problems and lack of motivation. There was no significant difference at baseline between the participants who completed the study and the participants who dropped-out in age, MMSE score, APOE genotype, and dementia type. For the cognitive domain scores, no differences were found, except for the domain of working memory in which the participants who dropped out performed significantly better at baseline than the participants who completed the study. In total 101 persons with dementia completed the study of whom 34 participated as controls. The participants from the two intervention groups $(N=67)$ were included in further analyses, 34 in the exergame group and 33 in the single aerobic group. The average age was 79.8 years, $54 \%$ were men, and $53.5 \%$ had Alzheimer's disease. Four patients improved significantly on global cognition ( $4.5 \%$ responders). For the separate cognitive domains, 9 participants improved significantly on executive function (13.4\%), 10 on processing speed $(14.9 \%), 4$ on episodic memory $(6.0 \%)$, and 3 on working memory $(4.5 \%)$.

The parameter estimates for all regression models are presented in Table 1. The regression model for global cognition did not show any significant relations. The regression model for episodic memory showed a significant negative relation between type of dementia and the RCI for episodic memory $\left(\mathrm{RCI}_{\mathrm{mem}}\right), B=-0.53(p=0.024)$, indicating that the $\mathrm{RCI}_{\text {mem }}$ in people with Alzheimer's disease is 0.53 units lower than the $\mathrm{RCI}_{\text {mem }}$ in people with other types of dementia. The regression model for working memory indicated two non-significant trends. First, there was a negative trend between APOE genotype

Table 1

Multiple regression model predicting the change in global cognitive function, episodic memory, working memory, executive function and processing speed (reliable change index)

\begin{tabular}{|c|c|c|c|c|}
\hline & B & SE B & $\beta$ & $\mathrm{p}$ \\
\hline \multicolumn{5}{|c|}{ Global cognition model $\left(R^{2}=0.089\right)$} \\
\hline Constant & -0.91 & 1.32 & & \\
\hline Age & 0.02 & 0.02 & 0.16 & 0.234 \\
\hline MMSE score & 0.01 & 0.03 & 0.04 & 0.762 \\
\hline$A P O E$ genotype & -0.19 & 0.19 & -0.12 & 0.340 \\
\hline Type of dementia & -0.26 & 0.19 & -0.17 & 0.164 \\
\hline \multicolumn{5}{|c|}{ Episodic memory model $\left(R^{2}=0.085\right)$} \\
\hline Constant & 1.89 & 1.66 & & \\
\hline Age & -0.01 & 0.02 & -0.08 & 0.549 \\
\hline MMSE score & -0.02 & 0.04 & -0.06 & 0.655 \\
\hline$A P O E$ genotype & -0.18 & 0.24 & -0.09 & 0.469 \\
\hline Type of dementia & -0.53 & 0.23 & -0.29 & $0.024 *$ \\
\hline \multicolumn{5}{|c|}{ Working memory model $\left(R^{2}=0.175\right)$} \\
\hline Constant & -2.41 & 1.35 & & \\
\hline Age & 0.03 & 0.02 & 0.23 & 0.075 \\
\hline MMSE score & 0.03 & 0.03 & 0.13 & 0.285 \\
\hline$A P O E$ & -0.39 & 0.20 & -0.24 & 0.053 \\
\hline Type of dementia & 0.11 & 0.19 & 0.07 & 0.563 \\
\hline \multicolumn{5}{|c|}{ Executive function model $\left(R^{2}=0.096\right)$} \\
\hline Constant & -3.40 & 2.32 & & \\
\hline Age & 0.01 & 0.03 & 0.04 & 0.783 \\
\hline MMSE score & 0.13 & 0.05 & 0.31 & $0.017 *$ \\
\hline$A P O E$ genotype & 0.04 & 0.34 & 0.01 & 0.916 \\
\hline Type of dementia & 0.19 & 0.32 & 0.07 & 0.558 \\
\hline \multicolumn{5}{|c|}{ Processing speed model $\left(R^{2}=0.111\right)$} \\
\hline Constant & 0.01 & 1.78 & & \\
\hline Age & 0.03 & 0.02 & 0.22 & 0.095 \\
\hline MMSE score & -0.09 & 0.04 & -0.28 & $0.028 *$ \\
\hline$A P O E$ genotype & 0.15 & 0.26 & 0.07 & 0.567 \\
\hline Type of dementia & -0.30 & 0.25 & -0.15 & 0.238 \\
\hline
\end{tabular}

B, unstandardized coefficient; SE B, standard error of unstandardized coefficient; $\beta$, standardized coefficient; $R^{2}$, the proportion of variance for a dependent variable that is explained by the predictors, ${ }^{*} p<0.05$. Levels: age in years; MMSE score between 0 and 30 ; APOE genotype: $0=\varepsilon 4$ non-carrier, $1=\varepsilon 4$ carrier; type of dementia: $0=$ non-Alzheimer; $1=$ Alzheimer. 
and the RCI for working memory $\left(\mathrm{RCI}_{\mathrm{wm}}\right), B=-0.39$ ( $p=0.053$ ), implying that the $\mathrm{RCI}_{\mathrm{wm}}$ in APOE $\varepsilon 4$ carriers is 0.39 units lower than the $\mathrm{RCI}_{\mathrm{wm}}$ in noncarriers. Second, we found a positive trend between age and the $\mathrm{RCI}_{\mathrm{wm}}, B=0.03(p=0.075)$, suggesting that when age increases with 1 unit, the $\mathrm{RCI}_{\mathrm{wm}}$ increases with 0.03 units. The regression model for executive function showed a significant positive relation between the MMSE score and the RCI for executive function $\left(\mathrm{RCI}_{\text {exe }}\right), B=0.13(p=0.017)$, indicating that when the MMSE score increases with 1 unit, the $\mathrm{RCI}_{\text {exe }}$ increases with 0.13 units. The regression model for processing speed showed a significant negative relation between the MMSE score and the RCI for processing speed ( $\left.\mathrm{RCI}_{\text {speed }}\right), B=-$ $0.09(p=0.028)$, indicating that when the MMSE score increases with 1 unit, the $\mathrm{RCI}_{\text {speed }}$ decreases with 0.09 units. Second, the model showed a positive trend between age and the RCI for processing speed ( $\left.\mathrm{RCI}_{\text {speed }}\right), B=0.03(p=0.095)$, suggesting that when age increases with 1 unit, the $\mathrm{RCI}_{\text {speed }}$ increases with 0.03 units.

\section{DISCUSSION}

In our study we found that only a minority of individuals with mild to moderate dementia showed significant and reliable improvements on global cognition $(4.5 \%)$ or on any of the cognitive domains (4.5-14.9\%) after a 12-week physical exercise intervention. Moreover, we found a smaller decline in episodic memory after the intervention in persons with non-Alzheimer disease compared to persons with Alzheimer's disease. We did not find significant associations between $A P O E \varepsilon 4$ status and global cognitive change or change in any of the domains. We observed a positive relation between baseline MMSE score and post-treatment change in executive function, while we found a negative relation between baseline MMSE score and post-treatment change in processing speed. These findings indicate that persons with less severe cognitive impairment at baseline show less cognitive decline in executive function after the intervention, while in contrast (and counter-intuitively), persons with more severe cognitive impairment at baseline show less decline in processing speed after the intervention.

A strength of this study is the use of RCI analyses, which contributes to a more personalized approach as it can help gain insight into which persons with mild to moderate dementia will benefit most from physi- cal activity interventions. There are also a number of limitations that need to be addressed. First, the fact that the RCI was not defined beforehand in our study protocol, but was added as post hoc analysis. Second, that we did not have one predefined primary outcome. Third, we only included the participants who completed the training and all measurements.

Overall, this study provides preliminary evidence that patient characteristics moderate the effect of physical activity on cognitive decline and that these effects differ per cognitive domain. Future research should focus on well-designed prospective studies with predefined RCI analyses to gain more insight into responder characteristics. These findings stress the need for a personalized approach when designing and analyzing interventions for persons with mild to moderate dementia to target those who benefit most from physical activity interventions.

\section{ACKNOWLEDGMENTS}

The authors thank Dr. William M. van der Veld for his statistical advice.

The project is funded by the Netherlands Organisation for Health, Research and Development (ZonMw), grant number 733050303. ZonMw did not contribute to the study design or to writing the manuscript.

Authors' disclosures available online (https:// www.j-alz.com/manuscript-disclosures/19-0606r2).

\section{REFERENCES}

[1] Groot C, Hooghiemstra AM, Raijmakers PGHM, van Berckel BNM, Scheltens P, Scherder EJA, van der Flier WM, Ossenkoppele R (2016) The effect of physical activity on cognitive function in patients with dementia: a meta-analysis of randomized controlled trials. Ageing Res Rev 25, 13-23.

[2] Forbes D, Forbes SC, Blake CM, Thiessen EJ, Forbes S (2015) Exercise programs for people with dementia. Cochrane Database Syst Rev, CD006489.

[3] Podewils LJ, Guallar E, Kuller LH, Fried LP, Lopez OL, Carlson M, Lyketsos CG (2005) Physical activity, APOE genotype, and dementia risk: findings from the Cardiovascular Health Cognition Study. Am J Epidemiol 161, 639-651.

[4] Rovio S, Kareholt I, Helkala E, Viitanen M, Bengt W, Tuomilehto J, Soininen H, Nissinen A, Kivipelto M (2005) Leisure-time physical activity at midlife and the risk of dementia and Alzheimer's disease. Lancet Neurol 4, 705-711.

[5] Hsu CL, Best JR, Davis JC, Nagamatsu LS, Wang S, Boyd LA, Hsiung GR, Voss MW, Eng JJ, Liu-Ambrose (2018) Aerobic exercise promotes executive functions and impacts functional neural activity among older adults with vascular cognitive impairment. Br J Sports Med 52, 184-191. 
[6] Karssemeijer EGA, Aaronson JA, Bossers WJR, Donders R, Olde Rikkert MGM, Kessels RPC (2019) The quest for synergy between physical exercise and cognitive stimulation via exergaming in people with dementia: a randomized controlled trial. Alzheimers Res Ther 11, 3 .

[7] Bruderer-Hofstetter M, Rausch-Osthoff A, Meichtry A, Munzer T, Niedermann K (2018) Effective multicomponent interventions in comparison to active control and no interventions on physical capacity, cognitive function and instrumental activities of daily living in elderly people with and without mild impaired cognition: a systematic review and network meta-analysis. Ageing Res Rev 45, 1-14.
[8] Karssemeijer EGA, Bossers WJR, Aaronson JA, Kessels RPC, Olde Rikkert MGM (2017) The effect of an interactive cycling training on cognitive functioning in older adults with mild dementia: study protocol for a randomized controlled trial. BMC Geriatr 17, 73

[9] Chelune GJ, Naugle RI, Lüders H, Sedlak J, Awad IA (1993) Individual change after epilepsy surgery: Practice effects and base-rate information. Neuropsychology 7, 41-52. 